\title{
Agronegocios
}

Artículo

\section{Valor económico de la prevención de enfermedades en animales en Costa Rica}

Economic value of animal deseases prevention in

Costa Rica

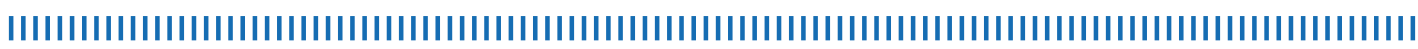

\section{Johanna Solórzano Thompson ${ }^{1}$ Javier Paniagua Molina² Tatiana Solano Pereira ${ }^{3}$}

Fecha de recepción: 04 de abril, 2020 Fecha de aprobación: 05 de junio, 2020

\section{Vol.6 N² 2 Julio- diciembre 2020}

Solórzano, J., Paniagua, J. y Solano, T. (2020). Valor económico de la prevención de enfermedades en animales en Costa Rica. Revista e-Agronegocios, 6(2). https: https://revistas.tec.ac.cr/index.php/eagronegocios/article/view/5081

DOI: https://doi.org/10.18845/ea.v6i2.5081

${ }^{1}$ Universidad de Costa Rica. Costa Rica. Docente en la Escuela de Economía Agrícola y Agronegocios. Correo electrónico: johanna.solorzano@ucr.ac.cr

2Universidad de Costa Rica. Costa Rica.Docente en laEscuela de Economía

Agrícola y Agronegocios. Correo electrónico: javier.paniagua@ucr.ac.cr. 3 Universidad de Costa Rica. Costa Rica. Docente en la Escuela de Economía Agrícola y Agronegocios. Correo electrónico: tatilu2892@gmail.com. 


\section{Resumen}

Todos los países pueden controlar o reducir el riesgo de enfermedades zoonóticas a través de la inversión pública en medidas preventivas como el control, la vigilancia y la atención de desastres. Costa Rica cuenta con un servicio veterinario oficial que cumple esta función y ha logrado reducir la prevalencia de algunas enfermedades de origen animal y efecto zoonótico. Esta investigación tiene como objetivo calcular el beneficio económico social de la prevención de las enfermedades en animales y su efecto en la salud pública en Costa Rica. Este estudio se realizó durante el periodo 2018 a través de reuniones de trabajo con médicos veterinarios privados y SENASA, médicos en salud humana, así como entrevistas con productores en diferentes fincas en todo el país. Además, se utilizaron fuentes secundarias para obtener estadísticas de prevalencias. Se aplicó la metodología de costos evitados y se calcularon los desembolsos que se evita el país ante la presencia de control y sin ésta. Los datos obtenidos demuestran un beneficio económico de más de US\$ 204.5 millones distribuidos en una composición del $21,9 \%$ en salud animal y $78,1 \%$ en salud humana. Existe un evidente impacto económico a nivel social, debido a la menor prevalencia de la enfermedad y por tanto la menor necesidad de combatirla tanto en fincas, casas de habitación, empleos, seguro social, entre otros.

Palabras clave: SENASA, costos evitados, beneficios económicos, enfermedades, zoonosis.

\section{Abstract}

Industrialized countries can control or reduce the risk of zoonotic diseases through public investment in preventive measures such as control, surveillance and disaster management. Costa Rica has an official veterinary service that fulfills this function and has managed to reduce the prevalence of some diseases of animal origin and zoonotic effect. This research aims to calculate the social economic benefit of disease prevention in animals and their effect on public health in Costa Rica. It was carried out during the 2018 period, through working meetings with private veterinarians and SENASA doctors, doctors in human health, as well as interviews with producers in different farms throughout the country. In addition, secondary sources were used to obtain prevalence statistics. The methodology of avoided costs was applied, and the disbursements that the country avoids in the presence of control and without it were calculated. The data obtained demonstrate an economic benefit of more than US\$204.5 million distributed in a composition of $21.9 \%$ in animal health and $78.1 \%$ in human health. There is an obvious economic impact at the social level, due to the lower prevalence of the disease and therefore the lower need to fight it both in farms, houses, jobs, social security, among others..

Key words: SENASA, avoided costs, economic benefits, diseases, zoonosis.. 


\section{Introducción}

En los últimos 60 años muchos países han controlado o eliminado con éxito las enfermedades zoonóticas mediante inversiones públicas costosas que facilitan intervenciones coordinadas, que incluyen "pruebas y sacrificios", prohibiciones de alimentación, vacunación masiva de animales domésticos y vida silvestre, educación sanitaria y pasteurización de la leche. Estos son métodos altamente efectivos para eliminar enfermedades zoonóticas que requieren garantías operacionales, legales y financieras importantes (Narrod, Zinsstag, y Tiogco, 2012).

Mientras que los países industrializados pueden controlar o reducir el riesgo de enfermedades zoonóticas a través de la inversión pública en medidas preventivas, como la vigilancia y la compensación de los agricultores por las poblaciones animales sacrificadas en caso de un brote, los paises en desarrollo carecen de capacidad de diagnóstico, y la vigilancia de estas enfermedades no se reconoce como una colaboración de "una sola salud" entre la medicina veterinaria y la medicina humana (Narrod, Zinsstag, y Tiogco, 2012).

Costa Rica cuenta con un servicio veterinario oficial denominado Servicio Nacional de Salud Animal (SENASA), que se encarga de establecer las directrices sanitarias de vigilancia epidemiológica y control de enfermedades en las poblaciones animales de interés económico y de salud pública, mediante investigación, inspección, erradicación de enfermedades y plagas. Así mismo, se destaca su participación en atención de denuncias y capacitación a la población (Ley 8495, 2006).

De las funciones que cumple SENASA se desprende una serie de servicios que generan beneficios socioeconómicos para el usuario y favorecen indirectamente a la población, se evitan costos de desembolso por gastos médicos, pérdida de ingresos y efectos menos tangibles como el dolor, la incomodidad y la restricción en actividades de trabajo del ser humano (Dixon, 1998). También se contribuye a fortalecer la salud pública veterinaria, favoreciendo la economía del país.

Con el fin de abordar temas de interés en salud pública, salud animal y medio ambiente, se han aplicado diferentes metodologías. A través del análisis de regresión, Ostro (1994) estimó los coeficientes que se multiplican por los cambios en las concentraciones de contaminación ambiental y la población expuesta. En el año 1998 se utilizó la función dosis-respuesta para estimar los impactos en la salud al reducir la contaminación del aire en Yakarta, Indonesia. Posteriormente, con el enfoque del costo de la enfermedad, se procedió a estimar valores monetarios a los efectos sobre la salud y la voluntad de pago para reducir la mortalidad prematura (Dixon, 1998). En México se cuantificaron lo beneficios monetarios de la población si disminuyeran los niveles de contaminación y su efecto en el padecimiento de asma, tomando como costos directos los tratamientos médicos y como costos indirectos la merma en la producción de la sociedad, expresada en horas de trabajo perdidas como consecuencia de la enfermedad (Torillo, 2008). 
Para establecer un parámetro de comparación de costos, Salman, et al. (1990) calcularon el costo anual de las medidas preventivas y el costo anual de la enfermedad Brucelosis por animal (vacas). Los costos preventivos contemplaron inyecciones, medicamentos y servicios veterinarios, mientras que el costo asociado con la aparición de enfermedades incluyó pérdidas por muertes y sacrificios, producción agrícola, el costo veterinario para el tratamiento de animales enfermos y costos de medicamentos, mano de obra, eliminación de cadáveres y transporte. El valor del beneficio económico social fue el resultado de la opción sin medidas preventivas versus la opción con control de la enfermedad.

Con el objetivo de alentar a los agricultores a adoptar buenas prácticas de producción, los esquemas de salud pueden proporcionar aseguramiento de la producción, bienestar social, proteger la salud pública, reducir la dependencia de los productos farmacéuticos y facilitar la administración, así como mejorar la productividad o eliminar enfermedades específicas. Además, la mejor manera de medir el impacto económico es a través de las pérdidas evitables, utilizar métodos de simulación para superar la ausencia de información y considerar la incertidumbre en estimaciones de costos (Stott, 2003).

En Argentina se contemplaron los costos generados por el cumplimiento de las regulaciones y medidas sanitarias vigentes y la consecuente eliminación de la Brucelosis, para compararse con los beneficios económicos del productor. También se consideraron beneficios indirectos que no fueron evaluados económicamente, pero que son importantes de analizar, como son: mejoras en la detección de problemas sanitarios y de manejo, mejoras en la inserción del país en el comercio internacional, control y erradicación de enfermedades con impactos en la producción y la salud humana (Gallacher, 2010).

Para reducir los efectos de la Brucelosis en Chile se contemplaron acciones de vigilancia, prevención, control y saneamiento predial, así como acciones de apoyo para acreditación de laboratorios y médicos veterinarios privados. Esto permitió calcular el beneficio basado en la diferencia de pérdidas que se producen a partir de dos escenarios: a) el Estado toma medidas para erradicar la enfermedad en 10 años, y b) el Estado no lleva a cabo ninguna acción. La investigación concluyó sobre la necesidad de adoptar medidas para erradicar la enfermedad mediante financiamiento público (Lopetegui, 2010). En Costa Rica se realizó un estudio para el control de la Brucelosis enfocado en los costos probables de un programa de erradicación y en sus beneficios potenciales. Estos costos se pueden estimar con base en: a) costos reales históricos de un programa similar al que se quiere implementar, y b) presupuestos de costos en los que se incurrirá. Estos incluyen lo relativo a control (análisis serológicos, vacunación) y no a pérdida de valor genético por sacrificio anticipado de vientres animales (Gallacher, 2010).

Por otra parte, en Brasil se analizó el costo-beneficio de desarrollar o no un programa de prevención para la fiebre aftosa. Este estudio demostró la relevancia de una adecuada intervención pública dirigida a la estructura de servicios veterinarios oficiales y programas nacionales de salud animal, además del éxito de implementar un programa nacional de erradicación de esta fiebre (Souza, Dolieira, Nogueira, Machado, y Figueiredo, 2010). El análisis costo-beneficio 
del programa nacional de fiebre aftosa en Perú consideró los costos relacionados con identificación y control de focos de fiebre, vacunas y capacitaciones. Los beneficios se cuantificaron como las pérdidas evitadas en producción de leche y carne por la demora de salir al mercado y por mortalidad. Dicho análisis presenta resultados favorables, ya que el indicador financiero (tasa interna de retorno) resultó superior a la tasa de capital utilizada, beneficiando a pequeños y medianos productores (Valderrama, 2010).

En lo que respecta a la peste porcina clásica (PPC), en Honduras se realizó un análisis costo-beneficio para los productores de traspatio, ya que sobre estos impactó en mayor medida la PPC. Aunque los productores industriales podrian beneficiarse por la eventual apertura de mercados y ahorros en costos de vacunación (Gallacher, 2010). En Chile se realizó una evaluación económica del programa de control y erradicación de la tuberculosis bovina, tomando en cuenta las pérdidas evitadas y la certificación sanitaria de la producción de leche. Sin embargo, no se consideraron las pérdidas evitadas por mortalidad de bovinos, mastitis tuberculosa, fertilidad por metritis tuberculosa, costos de servicios veterinarios y tratamientos, vida útil de las vacas infectadas, impacto en salud pública y pérdida de material genético (Paredes, 2010).

Para Howe, Häsler, y Stärk (2012) las enfermedades animales generan problemas económicos con implicaciones veterinarias, y la manifestación más evidente es la pérdida de producción como una medida del valor perdido para la sociedad. Del mismo modo, el miedo de las personas a las zoonosis reduce el bienestar social. Para evitar dichas pérdidas se debe incurrir en costos que mitiguen las enfermedades, con recursos para la investigación, el diseño y la inversión en medidas de vigilancia e intervención, y su implementación por personal calificado. Si el esfuerzo de mitigación excede el óptimo económico del valor monetario de las pérdidas de producción evitadas, se deben tener en cuenta los valores monetarios y no monetarios obtenidos de otros beneficios.

En vista de que las enfermedades zoonóticas provocan afectaciones en la salud humana que repercuten en la economía de un país, se requiere valorar el beneficio que se deja de percibir por esta causa. En las primeras mediciones del impacto de una enfermedad zoonótica en la salud de las personas, sólo se estudiaba la mortalidad (Zeckhauser y Shepard, 1976; Alvis y Valenzuela, 2010). Posteriormente, se consolidaron los intentos previos para construir un indicador que resuma las medidas de mortalidad y morbilidad, y su principal utilidad se refiere a los estudios de carga de la enfermedad que permiten medir las pérdidas de salud que representan la mortalidad prematura y la discapacidad asociada a las enfermedades (Schopper, et al., 2000).

La inversión exitosa en el control de las zoonosis requiere una evaluación del costo de la enfermedad y la relación costo-efectividad de las intervenciones propuestas, además de la adaptación de las intervenciones al contexto local. Dado que el 70\% de la población rural pobre del mundo depende del ganado y de los animales de trabajo para su sustento, los animales no pueden quedar fuera de las soluciones. Además, hay impactos a nivel sectorial, como el de alimentación e insumos, que afectan otros sectores asociados de la economía. A través 
del análisis integrado, se puede estimar el costo social total de la enfermedad relacionando un modelo de transmisión animal-humano (Narrod, Zinsstag, y Tiogco, 2012). Por esta razón, la presente investigación tiene como objetivo calcular el beneficio económico social de la prevención de las enfermedades en animales y su efecto en la salud pública en Costa Rica.

Referente teórico

SENASA es una institución pública y un servicio veterinario oficial, creado en el año 2006 como un órgano de mínima desconcentración bajo la personalidad jurídica instrumental, que se encarga de establecer las directrices sanitarias de vigilancia epidemiológica y control de enfermedades en las poblaciones animales de interés económico y de salud pública (SENASA, 2020). Se entiende como salud pública la capacidad de alcanzar el potencial biológico y mental de la población, sin limitaciones por lesiones, incapacidad o enfermedad (OPS, 2002).

Por otro lado, se entiende por zoonosis aquellas enfermedades e infecciones que se transmiten de forma natural entre los animales vertebrados y el hombre, y viceversa. Algunos efectos negativos de la incidencia de estas enfermedades son altas tasas de morbilidad y mortalidad, repercusión económica en la productividad laboral, disminución del turismo en zonas afectadas, y reducción de la riqueza pecuaria y la producción de alimentos (Acha y Szyfres, 2001; Brown, 2001).

Para evitar o disminuir posibles pérdidas a causa de las zoonosis, se deberá incurrir en costos. Estos costos se conocen como costos evitados o inducidos, y parten del supuesto que, si las personas están dispuestas a incurrir en costos para evitar los perjuicios causados por la pérdida de algún servicio -en este caso los servicios ofrecidos por SENASA- entonces, estos servicios deben valer, al menos, el monto que la gente paga por ello (Cristeche y Penna, 2008). Es necesario considerar que estos servicios generan beneficios para la población, y en el caso de la valoración de beneficios de una determinada intervención (salud) o realización de una actividad, se pueden entender como los ahorros generados por los casos que hubieran tenido que desembolsar para ser tratados o atendidas las consecuencias generadas. Por lo tanto, la estimación de los beneficios es en esencia un análisis de costos (Mendoza, 2002).

\section{Metodología}

Según la Organización Mundial de la Salud Animal (OIE, por sus siglas en inglés), existen declaradas 117 enfermedades en animales, de las cuales el 35\% son zoonóticas para el ser humano y 27 tienen presencia en Costa Rica (Hutter, 2013). Estas se evaluaron con los expertos en epidemiología del SENASA, para determinar cuáles de ellas son las más relevantes en la salud pública, tomando en cuenta su efecto en la salud humana y animal, y se categorizaron en una escala Likert de 1 (muy poco importante) a 5 (extremadamente importante) según el grado de importancia.

La investigación se realizó en el año 2018 a través de reuniones de trabajo y paneles con mé- 
dicos veterinarios y médicos en salud humana, así como entrevistas con productores en diferentes regiones del país. Se utilizó la metodología de costos evitados como el resultado de los ahorros producidos al cuantificar los desembolsos en que se debería incurrir en presencia de las enfermedades, tanto para productores como instituciones de salud, con la premisa de ser tratadas o atendidas y sus consecuencias generadas, versus el control con baja o ninguna prevalencia ${ }^{1}$ de la enfermedad.

Con este método se busca cuantificar los costos de prevenir pérdidas asociadas a las enfermedades de los animales y las pérdidas de tratar a las personas y la reducción de su productividad por la probabilidad de zoonosis. El procedimiento de cuantificación consiste en la identificación de la frecuencia de los diferentes escenarios posibles y su costeo (Mendoza, 2002).

Se consideraron dos escenarios: 1) con control de la enfermedad tal y como se realiza en Costa Rica actualmente a través del SENASA; y 2) sin control de la enfermedad con el supuesto de que no exista ninguna institución encargada en el país para su respectivo control.

El beneficio económico se midió como el costo evitado por la presencia de las enfermedades y se calculó mediante la siguiente ecuación:

$$
Y=B A+B H
$$

Donde,

Y es el beneficio económico total medido a través del costo evitado de la enfermedad;

BA es el beneficio económico total medido a través del costo evitado de la enfermedad en animales;

$\mathrm{BH}$ es el beneficio económico total medido a través del costo evitado de la enfermedad en humanos.

Los beneficios económicos al controlar las enfermedades en animales, incluyen:

$$
B A=A+D+S
$$

$1 \quad$ Proporción de individuos que se encuentran enfermos al momento de evaluar el padecimiento en la población (Fajardo, 2017). 
Donde,

A es el beneficio económico representado por medio del costo evitado de la atención de la enfermedad;

D es el beneficio económico representado por el costo evitado del desecho ${ }^{1}$ de animales muertos;

S es el beneficio económico representado por medio del costo evitado de la atención de la enfermedad en animales susceptibles².

Fue necesario conocer el número posible de animales enfermos mediante la prevalencia con control o sin control, por lo que se investigaron los casos de mayor prevalencia que se han presentado en Costa Rica o en el mundo en ausencia de intervenciones sanitarias y, a su vez, se determinó la prevalencia en un país que sí realiza el control de la enfermedad. Estos animales enfermos necesitan incurrir en un costo de atención médica ${ }^{3}$, aunque existe la probabilidad de muerte y se incurre en un costo de desecho.

Los beneficios económicos de controlar esas enfermedades en salud humana están dados por:

\section{$B H=E+T+N+C$}

Donde,

E es el beneficio económico representado por medio del costo evitado de la atención de la población enferma;

T es el beneficio económico representado por medio del costo evitado del desecho de la población fallecida;

$\mathrm{N}$ es el beneficio económico representado por medio del costo evitado total por la incapacidad $^{4}$ de la población enferma;

C es el beneficio económico representado por medio del costo evitado por la erosión 5 del capital humano.

\footnotetext{
$1 \quad$ Entiéndase como el entierro de los animales muertos.

$2 \quad$ Individuo que es vulnerable a contraer la enfermedad.

3 Costo de consulta médica y medicamentos necesarios, y atención médica veterinaria.

$4 \quad$ Período de reposo con el fin de propiciar la recuperación de la salud.

$5 \quad$ Incapacidad permanente o prolongada del individuo que lo imposibilita a realizar actividades y generar ingresos.
} 
Se obtuvo los casos de mayor prevalencia que se han presentado en el mundo en ausencia de controles sanitarios y la prevalencia en un país que sí los tenga. Se calculó el costo del tratamiento necesario y el costo de la consulta, si la persona es diagnosticada con la enfermedad, obtenidos a través del trabajo con expertos en medicina general.

Además, ante la posibilidad de deceso, con revisión de literatura se determinó la probabilidad de letalidad de la enfermedad y posteriormente el costo de gastos funerarios. Finalmente, se tomó en cuenta que las personas económicamente activas que fallecen impactan la economía del país. Dicha pérdida se cuantificó a través de dos métodos. El primer método mediante el producto interno bruto per cápita anual (PPCA), donde se utilizó una edad promedio de 33 años (se obtuvo el porcentaje de la población para cada edad y se calculó un promedio general para los últimos ocho años) en Costa Rica (INEC, 2019), la tasa social de descuento que asciende a 8\% (Campos, Serebrisky, y Suárez, 2016) y el producto interno bruto per cápita anual de US\$11 300 (BCCR, 2018). Se calculó el PPCS capitalizado anualmente en US\$123 935 a través de la siguiente fórmula:

$$
C=R\left[\frac{1-(1+i)^{-n}}{i}\right]
$$

Donde,

R es el valor de la renta o pago periódico, en este caso el PPCA;

i es la tasa de la anualidad de 8\% (en términos reales);

n es el número de periodo de la capitalización utilizado de 33 años promedio.

El segundo método se calculó mediante la metodología del DALY, que consiste en la suma de años de vida perdidos (YLL) y los años de vida con discapacidad (YLDs). El impacto de las intervenciones en los DALY (es decir, los DALY evitados por una intervención) se calculó en dos escenarios: con y sin la intervención. Las fórmulas relevantes fueron descritas por Fox-Rushby y Hanson (2001) con datos de Murray y López (1996), como se muestra a continuación:

$$
\begin{gathered}
Y L L s[r, K, \beta]=\frac{K C e^{r a}}{(r+\beta)^{2}}\left\{e^{-(r+\beta)(L+a)}[-(r+\beta)(L+a)-1]-e^{-(r+\beta) a}[-(r+\beta) a-\quad \text { (5) }\right. \\
1]\}+\frac{1-K}{r}\left(1-e^{-r L}\right)
\end{gathered}
$$


Donde,

C es una constante:

K es el factor de modulación de la edad ponderada;

es el coeficiente de la función de edad ponderada;

a es la edad de defunción;

L es la esperanza de vida estándar a la edad de defunción;

r es la tasa de descuento social en términos reales.

Los datos utilizados en esta investigación son C igual a 0,1658; K igual a 1; a igual a 36; y L igual a 34,75 (Murray y López, 1996; Fox-Rushby y Hanso, 2001). Para r se utilizó la tasa de descuento social en términos reales, igual a 0,08 y igual a 35 considerada por los autores como la edad de madurez laboral.

Estos cálculos incorporan pesos relativos para la esperanza de vida, edad, tiempo futuro y discapacidad, y el costo efectividad por la pérdida de productividad por defunciones. Además, el costo efectividad por la pérdida de productividad por discapacidad está dado por:

$$
\begin{aligned}
Y L D S[r, K, \beta]= & D \frac{K C e^{r a}}{(r+\beta)^{2}}\left\{e^{-(r+\beta)(L+a)}[-(r+\beta)(L+a)-1]\right. \\
& \left.-e^{-(r+\beta) a}[-(r+\beta) a-1]+\frac{1-K}{r}\left(1-e^{-r L}\right)\right\}
\end{aligned}
$$

Donde,

C es una constante;

K es el factor de modulación de la edad;

es el coeficiente de la función de edad;

a es la edad de defunción;

L es la esperanza de vida estándar a la edad de defunción; 
r es la tasa de descuento social en términos reales;

D es el peso de la discapacidad.

De la misma manera, se utilizó C igual a 0,1658, K igual a 1; igual a 35; a igual a 35; L igual a 10; r igual a 0,08 y D igual a 0.60. También, la misma tasa de descuento social del 8\% y una inflación promedio del $4 \%$. Sustituyendo los factores mencionados anteriormente, se obtuvo un costo de oportunidad de la productividad perdida por persona de US\$105 388.

Aun cuando ambas metodologías de cálculo dan un valor similar, se tomó la decisión de utilizar el resultado del DALY, porque da un valor más conservador y se ha empleado históricamente en estudios relacionados con la valoración de salud (Zeckhauser y Shepard, 1976; Murray, et al., 1994; Schopper, et al., 2000; Fox-Rushby y Hanson, 2001; Alvis y Valenzuela, 2010).

\section{Resultados}

De la consulta a expertos veterinarios, abogados y administradores, se determinó que existen 13 enfermedades (Cuadro 1) que, ante la posibilidad de aparecer en Costa Rica, producirian un alto impacto en la salud animal y humana, y que actualmente son parte del control que ejercen las instituciones gubernamentales.

Cuadro 1. Resultados de la consulta a expertos y ponderación de las enfermedades más relevantes. Costa Rica, 2018.

\section{Enfermedad}

Encefalopatía espongiforme bovina

Prurigo lumbar

Influenza aviar

Tuberculosis

Brucelosis

Rabia

Peste porcina clásica

Gusano barrenador

Enfermedad de Newcastle

Fiebre aftosa

Infestación por Aethina tumida (Escarabajo de las colmenas)

Cabeza amarilla

Virus de la Tilapia del lago

\section{Ponderación}

$100 \%$

$100 \%$

$100 \%$

$93 \%$

$93 \%$

$87 \%$

$80 \%$

$80 \%$

$80 \%$

$73 \%$

$73 \%$

$73 \%$

$73 \%$ 
En cada enfermedad se consultó el tratamiento para combatirlo, tanto a veterinarios como productores y médicos, con el fin de calcular los costos de combatir cada enfermedad si no existiera un debido control, así como el costo de desecho de animales muertos y el control de los susceptibles. De acuerdo a lo establecido en la Ecuación 2, los resultados son los siguientes:

Cuadro 2. Costo total por la enfermedad en los animales sin intervención. Costa Rica, 2018.

\begin{tabular}{lccc}
\hline Enfermedad & $\begin{array}{c}\text { Costo total } \\
\text { de anlmales } \\
\text { tratados }\end{array}$ & $\begin{array}{c}\text { Costo total del } \\
\text { desecho de } \\
\text { anlmales muertos }\end{array}$ & $\begin{array}{c}\text { Costo del } \\
\text { tratamlento de } \\
\text { animales susceptlbles }\end{array}$ \\
\hline Influenza aviar & $2,039,317$ & 21,526 & $18,406,215$ \\
Encefalopatia espongiforme & 27,667 & 37,628 & 304,341 \\
bovina & 192,496 & 261,795 & 571,482 \\
Tuberculosis & $6,970,028$ & 1,129 & - \\
Gusano barrenador & 657,826 & 39,470 & $4,610,015$ \\
Fiebre del Nilo Occidental & 609,789 & 228,061 & $2,547,066$ \\
Fiebre aftosa & 151,492 & 1,683 & $3,253,641$ \\
Enfermedad de Newcastle & 9,252 & 353 & $1,714,325$ \\
Rabia canina & 470,345 & 639,670 & 738,318 \\
Brucelosis & 211,044 & 28,702 & 27,474 \\
Encefalitis equina & 86,601 & 21,362 & 426,313 \\
Peste porcina clásica & 29,095 & 39,569 & 110,549 \\
Rabia bovina & $\mathbf{U S \$}$ & $\mathbf{U S \$ \mathbf { 1 , 3 2 0 , 9 4 6 }}$ & $\mathbf{U S \mathbf { 3 2 } , \mathbf { 7 0 9 , 7 3 8 }}$ \\
\hline Total & $\mathbf{1 1 , 4 5 4 , 9 5 4}$ & & \\
\hline
\end{tabular}

Ante un escenario donde el país no realiza ningún tipo de prevención de las enfermedades, se cuantifica un monto total de US\$ 45485637.59 para el tratamiento de animales enfermos, animales susceptibles a enfermar y para el debido desecho de los animales muertos. Lo anterior, sin incluir lo respectivo a producción y comercio que el país no percibiría al presentarse alguna de estas condiciones, ya que no se encontraba bajo los alcances de esta investigación.

De la misma manera, se costeó cada enfermedad tomando en cuenta una prevalencia -como se explicó en la metodología- debido al control que ejercen las autoridades en el país. Este costo disminuyó en comparación a un escenario sin intervención (Cuadro 3). El costo total en el que incurriría el país si se presentaran las enfermedades en animales en un escenario donde existe control es de US\$750 542, por lo que el costo evitado total (Ecuación 2) en la salud animal corresponde a US\$ 44735095.

No obstante, es importante reconocer la repercusión que existe en las personas sobre la presencia de estas enfermedades en el pais por su naturaleza zoonótica. En el Cuadro 4 se mues- 
tra el costo de cada enfermedad si no existiera en el país una institución como SENASA y la prevalencia de la enfermedad fuese alta.

Cuadro 3. Costo total por la enfermedad en los animales con intervención. Costa Rica, 2018.

\begin{tabular}{lccc}
\hline Enfermedad & $\begin{array}{c}\text { Costo total } \\
\text { de animales } \\
\text { tratados }\end{array}$ & $\begin{array}{c}\text { Costo total } \\
\text { del desecho de } \\
\text { animales } \\
\text { muertos }\end{array}$ & $\begin{array}{c}\text { Costo de } \\
\text { tratamiento de } \\
\text { animales } \\
\text { susceptibles }\end{array}$ \\
\hline Influenza aviar & - & - & - \\
Encefalopatía espongiforme & 4,803 & 6,532 & 1 \\
bovina & 49,525 & 67,353 & 787 \\
Tuberculosis & - & - & - \\
Gusano barrenador & 18 & 1 & - \\
Fiebre del Nilo Occidental & - & - & - \\
Fiebre aftosa & - & - & 323.701 \\
Enfermedad de Newcastle & - & - & 21,934 \\
Rabia canina & 15,770 & 21,448 & 227,279 \\
Brucelosis & 380 & 52 & 6,883 \\
Encefalitis equina & 129 & 32 & 290 \\
Peste porcina clásica & 85 & 116 & 3.423 \\
Rabia bovina & US\$ 70,710 & US $\mathbf{9 5 . 5 3 4}$ & US\$584,298 \\
\hline Total & & & \\
\hline
\end{tabular}


Cuadro 4. Costo total por la enfermedad en personas sin intervención. Costa Rica, 2018.

\begin{tabular}{|c|c|c|c|c|}
\hline Enfermedad & $\begin{array}{l}\text { Total de } \\
\text { servicios } \\
\text { médicos }\end{array}$ & $\begin{array}{l}\text { Costo total por } \\
\text { fallecimiento }\end{array}$ & $\begin{array}{l}\text { Costo total de } \\
\text { incapacidad }\end{array}$ & $\begin{array}{l}\text { Erosión } \\
\text { del capital } \\
\text { humano }\end{array}$ \\
\hline Influenza aviar & 561,749 & $1,274.714$ & 132,208 & $80,632,389$ \\
\hline $\begin{array}{l}\text { Encefalopatía } \\
\text { espongiforme bovina }\end{array}$ & 43,873 & 863,685 & - & $54,632,610$ \\
\hline Tuberculosis & $4.560,706$ & 213.762 & $1,295,161$ & 13.521 .571 \\
\hline Gusano barrenador & 286,259 & - & - & - \\
\hline $\begin{array}{l}\text { Fiebre del Nilo } \\
\text { Occidental }\end{array}$ & 6,448 & 110 & 10,141 & 6,964 \\
\hline Fiebre aftosa & 16 & - & 89 & - \\
\hline $\begin{array}{l}\text { Enfermedad de } \\
\text { Newcastle }\end{array}$ & - & - & - & - \\
\hline Rabia canina & 1.552 & 18.571 & 83 & $1,174,692$ \\
\hline Brucelosis & 52,827 & 2,145 & 32,700 & 135,671 \\
\hline Encefalitis equina & 69,800 & 13.595 & 111,672 & 859.948 \\
\hline Peste porcina clásica & - & - & - & - \\
\hline Rabia bovina & 82 & 977 & 4 & 61,826 \\
\hline Total & US\$ $5,583,313$ & US\$ 2,387,559 & US\$ $1,582,060$ & US\$ $151,025,672$ \\
\hline
\end{tabular}

Con respecto del Cuadro 4, si el país no realiza ningún tipo de prevención de las enfermedades debe costear un monto de US\$160,578,604 (suma de todos los costos del cuadro anterior) para la atención médica de las personas enfermas, el costo de la incapacidad, el costo por fallecimiento y, además, el costo en que incurre el país por perder a personas que son económicamente activas. También se calculó el costo de cada enfermedad tomando en cuenta una prevalencia baja o nula debido al control que se da en el país, lo que resultó en una disminución del costo total en que incurriría el país si se presentaran las enfermedades en seres humanos (Cuadro 5). Por lo tanto, en un escenario donde existe control, el costo total es de US\$ 723484 y el costo evitado (Ecuación 3) en la salud humana corresponde a US\$ 159 855. 
Cuadro 5. Costo total por la enfermedad en personas con intervención. Costa Rica, 2018.

\begin{tabular}{lcccc}
\hline Enfermedad & $\begin{array}{c}\text { Total de } \\
\text { serviclos } \\
\text { médlcos }\end{array}$ & $\begin{array}{c}\text { Costo total } \\
\text { por } \\
\text { falleclmlento }\end{array}$ & $\begin{array}{c}\text { Costo total } \\
\text { de } \\
\text { Incapacldad }\end{array}$ & $\begin{array}{c}\text { Eroslón } \\
\text { del } \\
\text { capltal humano }\end{array}$ \\
\hline Influenza aviar & - & - & - & - \\
Encefalopatia espongiforme & 274 & 5.398 & - & 341,454 \\
bovina & 63,343 & 2,969 & 17,988 & 187,800 \\
Tuberculosis & - & - & - & - \\
Gusano barrenador & - & - & - & - \\
Fiebre del Nilo Occidental & - & - & - & - \\
Fiebre aftosa & - & - & - & - \\
Enfermedad de Newcastle & 58 & 696 & 3 & 44,051 \\
Rabia canina & 13,463 & 547 & 8,334 & 34,577 \\
Brucelosis & 11 & 2 & 18 & 139 \\
Encefalitis equina & - & - & - & - \\
Peste porcina clásica & 3 & 37 & - & 2,318 \\
Rabia bovina & US\$ 77,153 & US\$ 9,649 & US\$ 26,344 & US\$ 610,339 \\
\hline Total & & & & \\
\hline
\end{tabular}

Para dar solución a la Ecuación 1 con los datos anteriores, el beneficio económico del costo evitado es de US\$ 204590 215.64, siendo la influenza aviar la enfermedad que genera un mayor beneficio al país debido a su control, seguido de la encefalopatía espongiforme bovina (Cuadro 6). 
Cuadro 6. Beneficio económico del costo evitado según enfermedad. Costa Rica, 2018.

\begin{tabular}{|c|c|c|c|c|}
\hline Enfermedad & $\begin{array}{l}\text { Costo sin } \\
\text { control }\end{array}$ & $\begin{array}{c}\text { Costo con } \\
\text { control }\end{array}$ & $\begin{array}{l}\text { Beneflclo } \\
\text { económico }\end{array}$ & $\begin{array}{c}\text { Participación } \\
\text { porcentual }\end{array}$ \\
\hline Influenza aviar & $103.068,119$ & - & $103,068,119$ & $50,4 \%$ \\
\hline $\begin{array}{l}\text { Encefalopatia } \\
\text { espongiforme bovina }\end{array}$ & 55.909 .805 & 358,462 & 55.551 .343 & $27,2 \%$ \\
\hline Tuberculosis & $20,616.973$ & 389.766 & $20,227,207$ & $9.9 \%$ \\
\hline Gusano barrenador & $7,257,416$ & - & $7,257,416$ & $3.5 \%$ \\
\hline $\begin{array}{l}\text { Fiebre del Nilo } \\
\text { Occidental }\end{array}$ & 5.330 .974 & 19 & 5.330 .954 & $2,6 \%$ \\
\hline Fiebre aftosa & $3.385,022$ & - & $3.385,022$ & $1,7 \%$ \\
\hline $\begin{array}{l}\text { Enfermedad de } \\
\text { Newcastle }\end{array}$ & $3.406,816$ & 323.701 & $3,083.115$ & $1,5 \%$ \\
\hline Rabia canina & $2,918,828$ & 66.743 & $2,852,085$ & $1,4 \%$ \\
\hline Brucelosis & $2,071,677$ & 321,418 & $1,750,259$ & $0,9 \%$ \\
\hline Encefalitis equina & $1,322,236$ & 7,484 & $1,314,751$ & $0,6 \%$ \\
\hline Peste porcina clásica & 534,276 & 451 & 533,825 & $0,3 \%$ \\
\hline Rabia bovina & 242,102 & 5.982 & 236,120 & $0,1 \%$ \\
\hline Total & $\begin{array}{c}\text { US\$ } \\
206,064,242 \\
\end{array}$ & US\$ 1,474,026 & US\$ $204,590,216$ & $100,0 \%$ \\
\hline
\end{tabular}

El beneficio económico calculado presenta un importe mayor en la salud humana (Cuadro 7) al considerarse los tratamientos, las incapacidades temporales y permanentes que podria ser más costoso que en animales, por el efecto zoonótico.

Cuadro 7. Composición del beneficio económico del costo evitado total. Costa Rica, 2018.

\begin{tabular}{lcc}
\hline & $\begin{array}{c}\text { Beneficlo } \\
\text { económlco }\end{array}$ & $\begin{array}{c}\text { Particlpaclón } \\
\text { porcentual }\end{array}$ \\
\hline Beneficio económico en Salud Humana & $159,855,120$ & $78,1 \%$ \\
Beneficio económico en Salud Animal & $44,735,095$ & $21,9 \%$ \\
\hline Beneficio económico total & $\mathbf{2 0 4 , 5 9 0 , 2 1 6}$ & $\mathbf{1 0 0 , 0 \%}$ \\
\hline
\end{tabular}

\section{Discusión}

En el 2014, Costa Rica contaba aproximadamente con 2406418 hectáreas dedicadas a la producción agropecuaria, cerca del 47,1\% del territorio; además de otros espacios con algún tipo de producción animal en pequeña escala (INEC, 2015). Estas áreas son una fuente importante de trabajo que responden a la seguridad alimentaria del país y dinamizan las economías circundantes en consumo de insumos, alimentos y otros. La prevención de las enfermedades a 
través del Estado, evitan a éstos productores altos costos de mantenimiento y prevención. Tal y como lo indican Ramírez, Ríos y Moralez (2010), cuanta más cantidad de efectos positivos logren cuantificarse (costos evitados), mayor será el beneficio social generado por la inversión en prevención.

Gallacher (2010) concluyó que los estudios realizados en prevención de enfermedades en diferentes países de Latinoamérica, demuestran que los retornos asociados al control de enfermedades como la Brucelosis son superiores a los valores al retorno aceptable de proyectos públicos de inversión. Incluso en Costa Rica la prevención y reducción de la prevalencia tiene efectos económicos importantes en la producción. Asimismo, los programas de sanidad animal merecen atención prioritaria por parte de aquellos que toman decisiones presupuestarias, ya que pueden contribuir en forma positiva al desarrollo económico y al bienestar de la población (Gallacher, 2010).

Esta investigación mide como factor relevante el costo evitado del tratamiento tanto en animales como en personas, y se demuestra que los costos calculados pueden considerarse subestimados, ya que no es posible contar de manera pormenorizada con todos los costos implicados en cada una de las enfermedades tratadas en este documento (Torillo, 2008; Ramírez, et al., 2010).

Por sus efectos, es claro que el costo de evitar enfermedades en humanos es mayor al de evitarlas en animales. Sin embargo, esta metodología se utiliza bajo el supuesto de que las personas y animales reaccionan de la misma manera a una enfermedad y a un tratamiento. Es posible que la aplicación a comunidades específicas con patrones culturales específicos, genere alguna diferenciación en los resultados de costos y en algunos casos el costo evitado podría ser mayor.

\section{Conclusiones}

Con la metodología de costos evitados es posible cuantificar el beneficio económico y social que le genera a un país la prevención de las enfermedades en animales y en seres humanos. En Costa Rica, el SENASA cumple con la función de controlar, intervenir y prevenir la prevalencia de las enfermedades definidas como de mayor efecto tanto en animales como en la salud humana. El beneficio para la salud pública general se logra a partir de la cuantificación de la prevalencia de una enfermedad y su efecto económico de combatirla en los casos en que esta prevalencia sea alta (sin control) versus cuando sea baja (con control).

Esta investigación demuestra que el beneficio social en Costa Rica es de US\$ 204590 215.64, que se entiende como el costo en el que se deja de incurrir, debido a la menor prevalencia de enfermedades y, por lo tanto, la menor necesidad de combatirlas en fincas, casas de habitación, empleos, entre otros.

La inversión del Estado en este tipo de controles en animales y en la probabilidad de zoonosis, 
debe ser medida y publicada a la ciudadanía, ya que la prevención permite que entes privados y públicos eviten costos adicionales en sus operaciones. Además del efecto en la salud pública, existe un evidente impacto a nivel social que se extiende por encima de la propia enfermedad y que trasciende a la producción, el comercio y el turismo, y sus efectos deberían ser evaluados en futuras investigaciones.

En Costa Rica existe escasa información de carácter público que registre la prevalencia de enfermedades y los casos documentados de zoonosis, por lo que es necesario recurrir fuentes secundarias de información. Este estudio podría brindar resultados más exactos acerca de regiones específicas en el país para las estimaciones de las prevalencias de enfermedades.

Este tipo de investigaciones contribuyen tanto para dar a conocer los beneficios que percibe la población del país, así como para que el Estado reconozca el valor de su inversión. Por otra parte, la información obtenida permite la colaboración y la transferencia de resultados entre países en términos de politicas en sanidad animal.

\section{Literatura citada}

Acha, P., Szyfres, B. (2001). Zoonosis y enfermedades transmisibles comunes al hombre y a los animales (Vol. III). Washington, Estados Unidos: Organización Panamericana de la Salud.

Alvis, N., Valenzuela, M. (2010). Los QALYs y DALYs como indicadores sintéticos de salud. Revista Médica de Chile, 138(2), 83-87. http://dx.doi.org/10.4067/S0034-98872010001000005

BCCR (Banco Central de Costa Rica). (2018). Indicadores económicos: Producción y empleo. San José, Costa Rica. https://www.bccr.fi.cr/

Brown, C. (2001). La importancia de las enfermedades emergentes para la salud animal, la salud pública y el comercio. Conf. OIE, 2001, 13-17. https://www.oie.int/doc/ged/D5206.PDF

Campos, J., Serebrisky, T., Suárez, A. (2016). Tasa de descuento social y evaluación de proyectos. Banco Interamericaco de Desarrollo. https://publications.iadb.org/en/publications

Cristeche, E., Penna, J. (2008). Métodos de valoración económica de los servicios ambientales. Estudios socioeconómicos de la sustentabilidad de los sistemas de producción y recursos naturales ( ${ }^{\circ}$ 3). Argentina: INTA. https://inta.gob.ar/sites/default/files/script-tmp-metodos_doc_03.pdf

Dixon, J.A. (1998). The economic valuation of health impacts. The World Bank. https://www. preventionweb.net/files/1072_EconomicValuationofHealthImpacts1998.pdf

Fajardo, A. (2017). Measurement in epidemiology: prevalence, incidence, risk, impact measures. Revista Alergia México, 64(1), 109-120. http://www.revistaalergia.mx/ojs/index.php/ 
$\mathrm{ram} /$ article/view/252/448

Fox-Rushby, J., Hanson K. (2001). Calculating and Presenting Disability Adjusted life years (DALYs) in Coste-Effectiveness Analysis. Health Policy and Planning, 16(3), 326-331. http://dx. doi.org/10.1093/heapol/16.3.326

Gallacher, M. (2010). Análisis costo-beneficio de sanidad animal: Estudios de caso en Latinoamérica. Buenos Aires, Argentina: Organización Mundial de Sanidad Animal.

Howe, K., Häsler, B., Stärk, K. (2012). Economic principles for resource allocation decisions at national level to mitigate the effects of disease in farm animal populations. Epidemiol Infect, 141(1), 91-101. http://dx.doi.org/10.1017/So95026881200060X

Hutter, S. (2013). Informe sobre la situación sanitaria de Costa Rica. Sección de Vigilancia Epidemiológica, Heredia, Costa Rica: SENASA. http://www.senasa.go.cr/senasa/sitio/files/060114055646.pdf

INEC. (2015). VI Censo Nacional Agropecuario: Resultados generales. San José, Costa Rica: INEC. http://www.inec.go.cr/censos/censo-agropecuario-2014

INEC. (2019). Población: Series. San José, Costa Rica: INEC. https://www.inec.cr/poblacion/ temas-especiales-de-poblacion

Ley N 8495. Ley General del Servicio Nacional de Salud Animal. Diario Oficial La Gaceta, San José, Costa Rica, 16 de mayo de 2006.

Lopetegui, P. (2010). Evaluación de la estrategia planteada para un proyecto de erradicación de Brucelosis bovina en Chile. En Análisis costo-beneficio de sanidad animal: Estudios de caso en Latinoamérica (41-51). Buenos Aires, Argentina: Organización Mundial de Sanidad Animal.

Mendoza, P. (2002). Evaluación económica en Salud. San Marcos, Lima, Perú: Universidad Nacional Mayor de San Marcos.

Murray, C. (1996). The Global Burden of Disease. A comprehensive assessment of mortality and disability for diseases, injuries and risk factors in 1990 and projected to 2020. World Health Organization, The World Bank. https://apps.who.int/iris/handle/10665/41864

Murray, C., López, A., Jamison, D. (1994). The global burden of disease in 1990: summary results, sensitivity analysis and future directions. Bull World Health Organ, 72(3), 495-509. https://www.ncbi.nlm.nih.gov/pubmed/8062404

Narrod, C., Zinsstag, J., Tiogco, M. (2012). A one health framework for estimating the econo- 
mic costs of zoonotic diseases on society. Ecohealth, 9(2), 150-162. http://dx.doi.org/10.1007/ s10393-012-0747-9

OPS (Organización Panamericana de la Salud). (2002). La salud pública en las Américas: Nuevos conceptos, análisis del desempeño y bases para la acción. Washington, Estados Unidos: OPS. https://www.paho.org/hq/index.php?option=com_content\&view=article\&id=4036:\&lang=es

Ostro, B. (1994). Estimating health effects of air pollutants: A methodology with an application to Jakarta. Policy Research Working Paper 1301. Washington, Estados Unidos: The World Bank.

Paredes, L. (2010). Evaluación Económica del Programa de control y erradicación de la Tubercolosis Bovina (Chile). En Análisis costo-beneficio de sanidad animal: Estudios de caso en Latinoamérica (280-292). Buenos Aires, Argentina: Organización Mundial de Sanidad Animal.

Ramírez, C.L., Ríos, C.P., Morales, T. (2010). Estimación de costos inducidos derivados de la calidad del agua potable en Risaralda. Scientia et Technica, 44, 117-122. http://dx.doi. org/10.22517/23447214.1789

Salman, M.D., King, M.E., Wittum, T.E., Curtis, C.R., Odde, K.G., Mortimer, R.G. (1990). The national animal health monitoring system in Colorado Beef Herds: Disease rates and their associated costs. Preventive Veterinary Medicine, 8, 203-214. https://doi.org/10.1016/01675877(90)90012-7

Schopper, D., Pereira, J., Cuende, N., Alonso, M., Baylin, A., Ammon, C., Rougemont, A. (2000). Estimating the burden of disease in one Swiss canton: what do disability adjusted life years (DALY) tell us?. Int Epidemiol, 29(5), 871-877. http://dx.doi.org/10.1093/ije/29.5.871

SENASA. (2020). Institución: Historia. Servicio Nacional de Salud Animal, MAG. https://www. senasa.go.cr/

Souza, J., Dolieira, C., Nogueira, J., Machado, J., y Figueiredo, V. (2010). ACB do Programa Nacional de Prevencao e Erradicacao da Febre Aftosa (PNEFA). En Análisis costo-beneficio de sanidad animal: Estudios de caso en Latinoamérica (8-22). Buenos Aires, Argentina: Organización Mundial de Sanidad Animal.

Stott, A. (2003). Costs and benefits of preventing animal diseases: A review focusing on endemic diseases. Research and Development Division, Craibston Estate. https://www. webarchive.org.uk/wayback/archive/20170701074158/http://WwW.gov.scot/Publications/2005/01/20496/49533

Torillo, P.E. (2008). Estimación del beneficio económico en materia de salud por reduc- 
ciones de Ozono y PM10 en Mexicali: Un enfoque de costos evitadas. Colegio de la Frontera Norte, Tijuana, México. https://www.colef.mx/posgrado/wp-content/uploads/2009/06/TESIS-Torillo-Portilla-Esmeralda.pdf

Valderrama, W. (2010). Evaluación Económica del programa de control y erradicación de la fiebre aftosa en el Perú. En Análisis costo-beneficio de sanidad animal: Estudios de caso en Latinoamérica (190-220). Buenos Aires, Argentina: Organización Mundial de Sanidad Animal.

Zeckhauser, R., Shepard, D. (1976). Where now for saving lives?. Law and Contemporary Problems, 40(4), 5-45. https://scholarship.law.duke.edu/lcp/vol40/iss4/2/ 